\title{
El significante como categoría económica \\ (Por un concepto lingüístico de valor de uso)
}

\section{(1) Ricardo Abduca}

IIDyPCA, Universidad Nacional de Río Negro, Bariloche, Argentina Correo electrónico: rabduca@unrn.edu.ar
Recibido

Octubre de 2018

Aceptado

Abril de 2019

doi: $10.34096 /$ cas.i49.5306

\section{Resumen}

Se examina el empleo del término "valor de uso" en diversas obras contemporáneas de antropología económica. Se encuentra que la expresión está usada en tres sentidos diferentes. Afirmo que los tres son aproximaciones parciales a una descripción del funcionamiento de los valores que emergen del uso: las entidades materiales con las que se construye la sociedad humana. Sostengo que esa triple forma de manifestación corresponde exactamente al descubrimiento que constituyó, desde 1950, al estructuralismo: la autonomía de lo simbólico, como registro específico, aunque vinculado a lo imaginario y lo real. Los atributos del concepto de valor de uso aquí sugerido sugieren paralelismos notorios con el de significante. A saber: arbitrariedad e indiferencia, carácter cualitativo, versatilidad, capacidad de formar cadena. Desde esta perspectiva, se destacan ciertos evidentes fenómenos contemporáneos, como el carácter inscripto de la mercancía moderna. Si antaño la inscripción se reducía a la materia monetaria, ahora en cambio casi todos los bienes de uso están inscriptos.

\section{Signifier as an economic category. (Toward a linguistic concept of use-value).}

\begin{abstract}
The article examines the use of the term "use value" in various contemporary works of economic anthropology, finding that the expression is used in three different ways. I maintain that these three meanings are but partial approximations to a description concerning the functioning of the values that emerge from use: the material entities from which human society is built. I argue that this triple manifestation corresponds exactly with the discovery that, since 1950, founded structuralism: the autonomy of the symbolic, as a specific register, although linked to the imaginary and the real. The attributes of the use value concept here proposed suggest notorious parallelism with that of signifier: its arbitrariness and indifference, its qualitative character, versatility, and capability to form signifying chains. From this perspective certain obvious
\end{abstract}

\section{Palabras clave}

Valor de uso; Significante; Antropología económica; Economía política; Lingüistica

\section{Key words}

Use value; Signifier; Economic Anthropology; Political Economy; Linguistics 
contemporary phenomena are outlined, such as the fact that goods we use nowadays actually have an inscription, while in the distant past only the money had inscriptions.

\section{O significante como categoria econômica. (Por um conceito lin- guístico do valor de uso)}

\section{Resumo}

Palavras-chave

Valor de uso; Significante; Antropologia econômica Economia política; Linguística
Examina-se a utilização do termo "valor de uso" em diversas obras contemporâneas da antropologia econômica. $\mathrm{O}$ autor acha que a expressão foi usada em três modos diversos. Os três são aproximações parciais a uma descrição do funcionamento dos valores que emergem do uso: as entidades materiais da construção da sociedade humana. Essa tríplice forma de manifestação corresponde exatamente o descobrimento que constituiu, desde 1950, ao estruturalismo: a autonomia do simbólico, como registro especifico, vinculado, contudo, ao imaginário e ao real. É examinado o paralelismo dos atributos de dois conceitos, de valor de uso e o de significante. Os atributos do conceito de valor de uso que é sugerido aqui têm paralelismos notáveis com o de significante. Isso é: Arbitrariedade e indiferença. Caráter qualitativo. Versatilidade. Capacidade de formar cadeia. Desde esta perspectiva tentamos pensar evidentes fenômenos contemporâneos, como o caráter inscrito de quase todos os bens de uso de hoje em dia; no passado remoto, em vez disso, essa inscrição só era própria das moedas.

\section{Antecedentes. ¿Un concepto perimido?}

¿Hablar de valor de uso, todavía? ¿Qué ganamos con eso? El término forma parte de los conocimientos básicos de cualquier persona que ha hecho cursos básicos de economía y de ciencias sociales: lejos está de ser un término inusual. No obstante, o bien se lo emplea con poca frecuencia en la argumentación - salvo para referirse a autores clásicos (por ejemplo: Robotham, 2005); o directamente brilla por su ausencia, como es el caso de un trabajo de síntesis reciente, posiblemente la primera monografía dedicada a la historia de la antropología económica (Hahn y Hart, 2011). O sea que la expresión sería, o una pieza histórica, o bien algo cuyo sentido es claro y del cual no hay más que hablar. Si es así, ¿qué más puede decirse?

Sin embargo, puede que haya todavía bastante por decir. En algunas síntesis del estado del arte de la antropología económica, el término se emplea con cierta frecuencia (Narotzky, 1997). Quiero destacar que la expresión no sólo se utiliza todavía en los estudios de antropología económica contemporáneos, sino que se lo hace de un modo diverso, a veces equívoco. Creo que esa dispersión de sentidos tiene razón de ser, pero tendría que emplearse de modo más controlado.

El término "valor de uso" aparece en la literatura contemporánea de los países centrales en tres sentidos diferentes.

a. Las características físicas de un bien de consumo (o de uso) que se oponen a sus características sociales. Raras veces se habla explícitamente de características físicas (por ejemplo, Robotham, 2005). Para Chris Gregory, el concepto ya clásico designa a las características intrínsecas de un bien, frente a las extrínsecas, propias del valor de cambio (Gregory, 1982). 
b. La valoración subjetiva de la utilidad (Menger, 1996; Jevons, 2013). ${ }^{1}$ No puedo comentar a estos dos autores por razones de espacio. Pero veremos que este punto de vista subjetivo es también el de Jean Baudrillard, para quien el valor de uso es el significado de la mercancía: su elemento subjetivo o "parte ausente" del signo (Baudrillard, 1972).

c. Un índice simbólico (Sahlins, 1972; Taussig, 1980; Chevalier, 1982; Roseberry, 1993). En el caso de Marshall Sahlins, cuando decía que el modo de producción doméstico no se caracteriza por tener "producción para el uso" sino por su "producción de valores de uso" (Sahlins, 1972, p. 87). En cierto sentido también Gregory, para quien el rol de valor de uso se puede ver como una etapa de la vida social del objeto: los objetos "sean dones, mercancías o valores de uso se vuelven bienes" en el acto de consumo (Gregory, 1997, p. 81). Aquí, "valor de uso" describe a un tipo de sociedad, o un tipo de objeto que no es don ni mercancía. Esta descripción presenta problemas - por lo pronto, porque toda sociedad, empezando por la capitalista, produce valores de uso--.

Otra dificultad es cuando se caracteriza por ausencia. Como el comentario de Gregory (al pasar, por cierto) acerca de que el brazalete decorado que funciona en el sistema kula "no tiene valor de uso" (Gregory, 1997, p. 83). Si es así, ¿cómo puede funcionar en sociedad? ¿No será que tiene otro valor de uso?

Sostengo aquí que la intensión del concepto "valor de uso" tal como aparece disperso en distintos textos de Marx, presenta isomorfismos con las notas intensivas del concepto de significante, tal como aparece en la obra de los lingüistas a partir de Saussure. Fui elaborando lentamente este punto de vista. Su primera versión fue expuesta hace ya algún tiempo en revistas argentinas (Abduca, 2004 y 2005).

Afirmo que los tres sentidos son aproximaciones parciales a una buena descripción del funcionamiento efectivo de los procesos de uso. Es decir, del empleo de las entidades materiales con las que se construye una sociedad humana. No obstante, no quiero decir que una descripción correcta consista en una mezcla empirista de "un poquito de cada uno" de los tres aspectos, sino que los tres puntos de vista toman aspectos parciales de la forma de manifestarse del uso como fenómeno. Y que en esa forma de manifestación, no sólo se verifica un cuerpo sensible y una imagen subjetiva, sino también el descubrimiento que constituyó, desde 1950, al estructuralismo: la autonomía de lo simbólico, como registro específico, irreductible a la secular dualidad que, con nombres cambiantes, recorre los tratados filosóficos y teológicos: lo real y lo imaginario, lo objetivo y lo subjetivo, lo material y lo ideal. El manifiesto de Claude Lévi-Strauss en su lectura de Marcel Mauss en 1950 signó la emergencia del paradigma estructural, con sus tres registros: real, imaginario y simbólico. Lo que me interesa mostrar aquí es que en el fenómeno social complejo designado por el concepto de valor de uso se anudan dimensiones corporales, imaginarias y simbólicas (Lévi-Strauss, 1980).

Es decir: el valor de uso funciona en tanto cuerpo, si y solo si es un cuerpo, y a partir de propiedades que se pueden verificar sensorialmente: se trata de un vínculo con el cuerpo humano. El uso es un nombre para un momento del recorrido de una vida humana.

Además, el uso es también un fenómeno de valoración subjetiva, es creencia en la utilidad, es ponderación subjetiva, deseo, convicción de la necesidad de empleo de un objeto, imposibilidad de sustitución.

Tercero, el valor de uso es también una marca del funcionamiento de una estructura. Existe como momento de actualización de un orden simbólico, tal como el habla [parole] actualiza un sistema lingüístico [langue].
1. La novedad de estos autores, difundidos a partir de 1871, fue el apostar a medir la valuación subjetiva con métodos de cálculo integral (Menger, 1996; Jevons, 2013). He comentado a estos autores en otro lugar (Abduca, 2010). 
2. Aristóteles, La política, I, $1257 \mathrm{a}$ (1959, p. 40); Marx 198ob; versión en alemán, $1980 \mathrm{c}$-luego en $E l$ capital también insiste en esa lectura (1981).

En todas las referencias a la obra de Marx cito según la versión más asequible en castellano y luego según la edición alemana utilizada; ambas versiones están en las referencias bibliográficas al final del artículo. Aclaro también que en todas las citas, si hay palabras resaltadas en cursiva, el destacado corresponde al autor del texto citado.*
He explorado hace tiempo una lectura en tres dimensiones del concepto de valor de uso. Lejos de cambiar de idea, he insistido con el tema, pues creo que este enfoque tiene una de las virtudes que debe reclamársele a una perspectiva teórica, por más que sea, por supuesto, problemática y discutible: desarrollabilidad. Esta posibilidad creativa es la que permite que insistamos en lecturas de autores siempre inolvidados y siempre pasados de moda: Claude Lévi-Strauss, Ferdinand de Saussure, Karl Marx.

Se ha dicho que fue Marx quien, antes que Sigmund Freud, descubrió el síntoma. Sin pretender verificar esa afirmación, creo que ofrezco aquí una lectura en la que los textos maduros de Marx exhiben el despliegue de un significante (denominado Gebrauchswert, valor de uso). Ese despliegue muestra aspectos tanto corporales como imaginarios y simbólicos.

En efecto, las tres dimensiones se verifican en el empleo de "valor de uso" que aparece en los textos de Marx, aunque raras veces entre los marxistas.

Se dirá que se trata de una lectura retrospectiva, que introduce categorías de LéviStrauss o de Saussure en un corpus de textos que no fueron pensados en esos términos. Así leyó el mismo Marx a Aristóteles, pues introdujo el concepto contemporáneo de valor de uso (en particular el suyo propio, acuñado en los Grundrisse con el vocabulario de las traducciones alemanas de Adam Smith), en las citas griegas de La política. ${ }^{2}$ En efecto, cuando Marx escribe "valores de uso de la sandalia", interpreta con fuerza lo que literalmente es "usos de la sandalia” (hypodématos chrếseis). Más aún, como mostraré, es la forma de manifestarse de los bienes de uso lo que autoriza esta lectura. Es por eso que la exposición de textos maduros de Marx (El capital y otros) muestra estos tres aspectos. Sin embargo en el empleo del término después de Marx estas tres dimensiones generalmente no se ven juntas; en particular escasea el sentido de valor de uso como cuerpo.

Este enfoque busca respuestas alternativas a problemas planteados tiempo atrás por Baudrillard, quien hacia 1972 buscaba dar cuenta del peso de la cuestión del valor de uso en momentos en que el consumo (la adquisición de mercancías, y su empleo posterior) había cobrado una complejidad y una autonomía impensables un siglo atrás.

En términos estrictos, no creo que haya sido formulado por ningún autor que conozca. En el collage que hizo Baudrillard, a saber: entre valor de cambio y significante, y entre valor de uso y significado, yo he visto un problema, no una solución. No sé si lo he resuelto, pero he intentado dejarlo más claro.

Los obstáculos para desarrollar este punto de vista han sido varios. Uno es el hecho de que, fuera del ámbito estrictamente lingüístico, el concepto de significante ha sido usado y desarrollado, además del caso del análisis mitológico de Lévi-Strauss, sobre todo por el psicoanálisis lacanista. De los planteos que conozco, los más cercanos a mis argumentos han sido los expuestos por Jean-Joseph Goux hace un buen tiempo: cómo funciona un significante mayor como el dinero, leído en los términos de los tres registros lacanianos - real, imaginario, simbólico- (Goux, 1973). A pesar de todo su potencial, análisis como los de Goux, abocados a buscar las mediaciones directas entre el significante dinero y el significante falo, tienen el obstáculo del hiato que se produce entre dos niveles de análisis distintos, el del mundo prosaico material cotidiano y el del inconsciente.

Otro tipo de obstáculo es el peso del punto de vista de Marx: el modo en que está planteada su teoría del fetichismo. El misterio [Geheimnis] del carácter fetichista de la mercancía está en su forma misma: carácter místico del valor de cambio. Distinto es el caso del valor de uso:

\footnotetext{
las citas en español referen bro primero (vols. 1-3) de la edición de Siglo XXI. En esa edición, los 3 volúmenes correspondientes al libro primero, mantiene continuidad en la numeración de páginas. Por lo tanto, en el presente artículo se optó por referenciar a las páginas sin especificar el volumen.
} 
en cuanto valor de uso nada de misterioso [Mysteriöses] se oculta en [la mercancía], ya la consideremos desde el punto de vista de que merced a sus propiedades satisface necesidades humanas, o de que no adquiere esas propiedades sino en cuanto producto del trabajo humano. (Marx, 1981, p. 87; versión en alemán, 1962, p. 85)

Esto, a su vez, se vincula con otro aspecto de la teoría del fetichismo; Marx observó, al plantear la forma equivalente del valor, cómo "la forma natural de la mercancía se convierte en forma de valor"; se trataba de un quid pro quo, tomar una cosa por otra (Marx, 1981, p. 69). Esta misma figura, tomar una cosa por otra, se encuentra al analizar la forma fetichista de la forma de valor. Mediante este quid pro quo, las cosas se volvían "sensorialmente suprasensibles" [sinnlich übersinnliche] o sociales (Marx, 1981, p. 87; versión en alemán, 1962, p.85).

Es esta una definición de toda instancia simbólica. Con el análisis del fetichismo de la forma de valor, Marx describía la determinación simbólica en un tiempo en que no había mayor reflexión sobre la entidad de las estructuras lingüísticas. De hecho, en toda representación simbólica hay un quid pro quo. Es más, en ese mismo "tomar una cosa por otra" se revelan las formas sociales de la sustitución, los eslabones más fundamentales de una estructura. Al carecer de la problematización lingüística que tenemos desde el siglo XX, Marx desconfiaba de todo quid pro quo, pensando que una sociedad de productores directos: "una asociación de hombres libres que trabajaran con medios de producción colectivos" podía desvanecer "el reflejo religioso del mundo real" (Marx, 1981, p. 96).

Hoy, sin embargo, para entender mejor las condiciones de posibilidad de esa transparencia debemos tener en cuenta el peso de las instancias simbólicas e imaginarias, y no solo las materiales, en todos los órdenes de la vida social (registros simbólico e imaginario que Marx sí pudo ver bien, como mostró Goux al analizar el dinero). Las ciencias lingüísticas, que han abierto la vía para empezar a entender dichos dos registros imaginario y simbólico al lado de la dimensión estrictamente corporal, y que estaban relativamente subdesarrolladas en Occidente en tiempos de Marx - aun si se compara con el desarrollo en gramática y fonología al que se había llegado en la India más de 20 siglos atrás, con Pāṇini y Patañjali-conocieron luego un desarrollo impresionante, que es uno de los rasgos característicos del siglo XX, el cual no puede dejar de afectar, por supuesto, al estado de los problemas de la economía política crítica. El abordaje lingüístico permite ir, con Marx, más allá de Marx. Y observar esa instancia, la del valor de uso, que está más llena de "sutilezas ontológicas y teológicas" de lo que parecía hace un siglo y medio.

El planteo. Empecé a reflexionar sobre estos problemas gracias a reconocer lo incisivo del planteo temprano de Jean Baudrillard, no sin dejar por eso de desconfiar de los términos de ese planteo. El valor de uso, según Baudrillard, sería el significado de la mercancía. Los significantes del orden social serían los valores de cambio. El valor de uso es "expresión de toda una metafísica: la de la utilidad" (Baudrillard, 1972, p. 159). ${ }^{3}$ Las cadenas significantes, por su lado, forman parte de la trama del valor de cambio "el valor de cambio es al valor de uso como el significante al significado [...]; el valor de cambio es al significante como el valor de uso al significado" (Baudrillard, 1972, p. 143).

$\mathrm{Al}$ entender al valor de uso como un significado está entendiendo a aquel concepto como mera función de utilidad. Sin embargo, el empleo del término valor de uso, si bien es problemático, es mucho más rico y complejo que el de la mera función de utilidad de la economía neoclásica — que es, esta sí, utilidad abstracta, convertida en número-. Con el criterio de Baudrillard, el valor de uso (al ser significado) no sería sino la parte ausente del signo mercantil. En realidad, el valor de uso, en tanto cuerpo, es la parte presente de una relación social. Es, por ende, un significante: soporte de significación
3. En esta u otras citas extranjeras, salvo indicación contraria, es mi traducción. 
4. Las páginas que siguen, excepto las "Reflexiones finales", reformulan y amplían ligeramente un texto reciente (Abduca, 2018).

5. Las palabras destacadas en citas, en este caso como en todos los que siguen, son del autor del texto citado. o parte presente de un signo - si alguien dice "árbol” no tiene porqué haber presencia de un árbol: lo que está presente es el significante árbol.

El punto erróneo de Baudrillard - que brinda al mismo tiempo la posibilidad de superación - fue haber considerado a los valores de uso como significados, como función subjetiva de utilidad, y no como significantes, como cuerpos investidos de carga social —es decir, simbólica-. Otro problema de este planteo de Baudrillard es tomar una forma histórica determinada (aunque muy antigua, y pre capitalista, como la de mercancía) y compararla con una mucho más primordial, como la polaridad entre significante y significado.

No es que quiera decir que un valor de uso sea exactamente lo mismo que un significante lingüístico. Las entidades materiales tienen su diferencia específica con las entidades del idioma. Un cuerpo que funciona como valor de uso puede guardarse, tiene momentos de uso y de no uso. Tiene desgaste mínimo (un cuchillo, una máquina), o bien desgaste completo (el alimento, el combustible). En cambio, el significante lingüístico tiene otra relación con el principio de escasez. Nuestra capacidad de emitir frases es casi infinita; siempre se puede acudir a cualquier punto léxico del paradigma: toda lengua es un cuerno de la abundancia, al lado del mundo de las cosas.

Queda pendiente desarrollar las diferencias entre las notas intensivas del concepto de valor de uso con respecto a la comprensión del concepto de significante. Aquí destacamos los puntos en común, que muestran porqué pensamos en buscar construir un concepto que aúne a "significante" y "valor de uso".

a. Cuerpo: como significante y como valor de uso. "Valor de uso" designa a un cuerpo. No a una necesidad ni a una función

un valor de uso es un objeto externo, una cosa. Su utilidad no vuela por los aires. Sino que existe a través de las propiedades de los cuerpos de las mercancías [die Eigenschaften des Warenkörpers]. El cuerpo mismo de la mercancía, tal como hierro, trigo, diamante, etc., es pues un valor de uso o un bien. (Marx 1980a, p. 47; versión en alemán, 1962, p. 49)

En la primera versión de ese capítulo afirma "denominamos a la cosa útil misma o al cuerpo de la mercancía - tal como el hierro, trigo, diamante, etc. —, valor de uso, bien, artículo" (Marx, 1981, vol. 3, p. 972). En el primer planteo publicado, en 1859: "Esta existencia de la mercancía en cuanto valor de uso y su existencia natural palpable [natürliche handgreifliche Existenz], coinciden”. (Marx, 1980b, p. 9; versión en alemán, 1980c, p. 107).

Asimismo, se trata del vínculo del cuerpo humano con la naturaleza de la cual forma parte: "El valor de uso en cuanto tal expresa en primer término la relación del individuo con la naturaleza” (Marx, 1971b, p. 160, versión en alemán, p. 43).

El valor de uso es un cuerpo; entonces, como tal, es la parte presente de la cosa en tanto signo, es el nombre de la dimensión significante, es un cuerpo que soporta sentido. Así mirado, lo que Marx y Louis Althusser llamaron "soporte", Träger, debe ser reexaminado: como significante y como valor de uso. Hay que decir también que no hay tal cosa como "un significante", sino como momento de una cadena significante. El Träger funciona en cadena: recorrido (que puede llamarse metonímico) en el que se expresa la estructura. En tanto cadena, los soportes están presentando, actualizando (darstellen) a la estructura.

El significante es cuerpo, pero cuerpo que está representando. Es una presencia que alude a algo que no está presente (el significado). El valor de uso no corresponde, en 
principio, a una u otra carencia. No se vincula naturalmente a algo útil. No es una idea otorgada a priori por un sujeto calculador. Pertenece, en principio, al orden de la cosa extensa. Como el significante, es una cosa extensa ordenada en secuencia sintagmática, en relaciones que "se desarrollan en la extensión, tienen como soporte a la extensión" (Saussure, 1993, p. 129).

b) Arbitrariedad. Indiferencia. Los fenómenos descritos con la expresión "significante", al igual que los que son descritos con la expresión "valor de uso", son arbitrarios o indiferentes a priori, a posteriori no lo son.

El signo es “inmotivado, es decir, arbitrario con respecto al significado. [Signo, encarnado en el significante], no tiene ningún lazo natural con respecto a la realidad" (Saussure, 1997, p. 101). Saussure advertía también que el signo no unía a un nombre con una cosa: lo que suele decirse "nombre" es una imagen acústica, lo que suele decirse “cosa” es un concepto (Saussure, 1997, p. 98). ${ }^{6}$ Lévi-Strauss, retomando ideas de Benveniste, aclaró que el signo lingüístico puede ser arbitrario a priori, pero no a posteriori (Lévi-Strauss, 1977, p. 84).

También en Marx el carácter material de un soporte (Träger, es decir, valor de uso) puede considerarse indiferente (gleichgültig) o arbitrario (willkürlich). El oro pudo no haber sido, a priori, el soporte de la riqueza social. De hecho, en otras situaciones históricas, esas funciones han sido cumplidas por otros entes materiales, hasta por caracolas. A priori es inmotivado, indiferente, arbitrario que así sea. Pero a posteriori, hay una prensión del valor de uso, del significante, que hace que la materialidad que sirve de soporte al vínculo social no sea indiferente. Así, se verifica un carácter no arbitrario,

El material en que se expresa el símbolo no es de ningún modo indiferente [gleichgültig], por más variedad que haya mostrado históricamente. La evolución de la sociedad elabora, junto con el símbolo, también el material que cada vez lo expresa mejor y del que se trata luego de desvincularse; un símbolo, si no es arbitrario, requiere determinadas condiciones del material en el cual se representa. (Marx, 1971a, vol. 1, p. 70; versión en alemán, 1976 p. 79-80)

También se verifica un carácter arbitrario a priori. Esto se observa en el proceso laboral de valorización: "Al valor le es indiferente que su portador sea uno u otro valor de uso, pero le es imprescindible que su portador sea un valor de uso" (Marx, 1981, p. 228; versión en alemán, 1962, p. 203). También en la circulación:

su sustrato constituido por un determinado quantum de oro y plata importa poco, y por el contrario su cantidad está determinada en modo esencial, puesto que así el dinero es solamente un signo para una determinada cantidad de esta unidad. En cambio, en su determinación como medida (...) su sustrato material era esencial, mientras que su cantidad y su existencia eran fundamentalmente indiferentes (...) como simple medio de circulación y de cambio, la moneda de oro y de plata puede ser reemplazada por cualquier otro signo que exprese una determinada cantidad de su unidad, y así un dinero simbólico puede sustituir al dinero real porque el dinero material como simple medio de cambio es él también simbólico. (Marx, 1971a, vol. 1, p. 148; versión en alemán, 1976, p. 140).

c) Carácter cualitativo del valor de uso: Marx no es Smith. No hay cosas de más valor de uso que otras. Esa es la diferencia - pocas veces tenida en cuenta- no solo entre Marx y los neoclásicos marginalistas, sino entre Marx y los economistas clásicos. La concepción cuantitativa del valor de uso no comenzó con los marginalistas. Adam Smith señaló la paradoja del gran "valor en uso" del agua, frente al escaso "valor en uso" de los diamantes, siendo que estos objetos tienen, por el contrario, un "valor en cambio"
6. Al examinar las fuentes manuscritas del Cours, De Mauro -responsable de la edición crítica del texto- ve que "concepto" e "imagen acústica” no son sinónimos de significado y significante, como se lee en el texto impreso, sino que aquellos son sustituidos por estos a medida que Saussure precisa su idea de la lengua como sistema (Saussure, 1997, p. 438, n. 128). 
7. Efectivamente, tanto Smith como Ricardo escriben value in use, value in exchange. Recién con las traducciones de Das Kapital al inglés se generalizó el empleo de use-value, o de exchange value.

8. Entendiendo al funcionamiento del valor de uso como algo que no se mantiene sino en su desplazamiento; así, en cada momento de tal desplazamiento, cada significante se va encadenando con otro (Lacan, 1966a, 1966b). muy bajo y muy alto, respectivamente (por cierto, Smith parafraseaba las observaciones de Robinson en su isla).El diamante tiene poco valor en uso y mucho en cambio; el agua, por su parte, tiene poquísimo valor en cambio y muchísimo en uso. ${ }^{7}$ David Ricardo abrió sus Principios citando y comentando este razonamiento de Smith (Ricardo, 2004). En suma, hay cosas más o menos útiles.

En contraste, el concepto de valor de uso en Marx es absolutamente cualitativo. La única determinación cuantitativa de los valores de uso es la de la magnitud conmensurable entre materias idénticas - al comparar un sistema productivo capaz de lograr doce bolsas de trigo en vez de diez, etcétera-.

d) Indivisibilidad. Versatilidad. Es sabido que un significante es versátil. Puede entenderse - y usarse - siempre de otro modo. Estas propiedades del significante son bien conocidas por los psicoanalistas, que han estudiado el funcionamiento del chiste y del síntoma. En tanto significante del momento productivo, por su parte, las cosas siempre pueden soportar nuevos usos, nuevas conexiones, y es de este modo como el sujeto productivo es capaz de enriquecer y potenciar las fuerzas productivas. Un valor de uso no es, en su uso, divisible: medio automóvil no es un auto a medias; medio automóvil no es un auto. Medio lexema tampoco es un lexema.

Asimismo, un significante es versátil. Escuchemos a Marx: "El mismo valor de uso puede usarse de diferentes maneras. La suma de sus utilizaciones posibles [Die Summe seiner möglichen Nutzanwendungen] le da, sin embargo, su existencia como cosa con determinadas propiedades [Dasein als Ding mit bestimmten Eigenschaften]" (Marx, 1980b, p. 9-10; versión en alemán, 1980c, p.107-108).

Asimismo, esa posibilidad productiva es capaz de bricoler. El pedazo de madera concebido como pata de una silla puede ser usado, como veía Lévi-Strauss, como materia prima para fabricar otro artefacto. El objeto puede pivotear, como el significante lingüístico en el chiste.

e) Formar cadena. El sentido de cada momento aparece en el eslabón siguiente. Un tipo de encadenamiento fundamental es el de la perpetua metamorfosis del capital, que no existe sino encarnado en un tipo u otro de valor de uso. En maquinarias, en dinero, en productos ofrecidos a la venta. Una vez que el valor en potencia llegó al acto, le llega el turno al eslabón siguiente (de una materia prima a producto terminado, de producto terminado a producto vendido, etcétera).

"El significante" nunca es uno, es un momento de una cadena significante. El Träger, significante, funciona en la cadena en la que se expresa la estructura. En tanto cadena de soportes, Träger, están presentando, actualizando (darstellen) a la estructura. Un símbolo es un cuerpo, considerado como lugar-teniente: ocupa un lugar en una cadena de usos. Cada cosa que ocupa un lugar ha venido a sustituir a otra. Así, un proceso de trabajo puede ser considerado como un proceso de funcionamiento metonímico del significante. ${ }^{8}$

f) El intercambio como cadena significante. Subjetividad. La forma de valor, valor de cambio, es una relación entre dos valores de uso. El objeto funciona como significado para el comprador: es un lugar vacío en la cadena significante que le interesa establecer; el sentido de su adquisición es ubicarlo en una cadena significante en un momento determinado. Para el vendedor, funciona como significante: parte presente. En cada polo se verifica una relación subjetiva entre lo particular y lo formal. Cada uno llega a la transacción con un tipo de nudo entre un valor de uso particular que sobra y un valor de uso formal que falta. Llamo a esa relación una posición de sujeto. 
Se llama comprador a quien, con dinero concreto (valor de uso particular) en la mano, observa el cúmulo de mercancías y sopesa los valores de uso formales que le interesan (que lo constituyen como sujeto en tanto le hacen falta). Se llama vendedor a quien, con valores de uso particulares que atesora en su territorio, espera a cambio un dinero (valor de uso formal del dinero).

En la posición inicial, el capital, como comprador, carece de fuerza de trabajo. El trabajo vivo se le contrapone como valor de uso formal. La capacidad laboral, el trabajo vivo, como cuerpo, corresponde al valor de uso particular que el trabajador vende.

La analítica del valor de uso recorre páginas fundamentales de "El capítulo del Capital" de los Grundrisse (Marx, 1971a, vol. 1 y 2), donde se descubre y expone, por primera vez, la diferencia entre trabajo y fuerza de trabajo (llamada en estos textos Arbeitsvermögen, capacidad de trabajo), hallazgo que sería el cimiento de toda la obra posterior de Marx.

Así como el valor de uso no es sino el cuerpo de la mercancía, nuestro valor de uso (el de toda persona) no es, en principio, otra cosa que la "constitución corporal". Así, el trabajador "cambia su valor de uso por la forma universal de la riqueza" (Marx, 1971a, vol. 1, p. 225-226; versión en alemán, 1976, p. 206). Asimismo, "el trabajo no es tan solo el valor de uso enfrentado al capital, sino que es el valor de uso del capital mismo" (p. 238; versión en alemán, p. 218); "como valor de uso el trabajo existe únicamente para el capital" (p. 246; versión en alemán, p. 225). El trabajador usa medios de producción ajenos.

Más aún:

En cuanto esclavo, el trabajador tiene valor de cambio [...] como trabajador libre no tiene ningún valor, sino que sólo la disposición sobre su trabajo, por el intercambio que se opera con él mismo, tiene valor [...] [Al capital industrial] el trabajo tiene que contraponérsele como puro valor de uso (Marx, 1971a, v. 1, p. 232; versión en alemán, 1976, p. 211)

La reflexión sobre el uso, como vemos, permite precisar mejor la cuestión de la esclavitud. Más aún, permite ayudar a plantear un más allá de las formas mercantiles de pensar lo social. Esto puede leerse a la luz de la acumulación de conocimientos antropológicos e históricos, y de nuevas preguntas, como las que han permitido a David Graeber mostrar una génesis del lazo social desigual no a partir del trueque sino de la esclavitud y la deuda. La secular desigualdad de la especie humana no provendría del uso de la naturaleza sino del uso de otro ser humano en los mecanismos de deuda y esclavitud (Graeber, 2011).

g) Formal y particular. El valor de uso en Marx sería "siempre concreto y particular", (Baudrillard, 1972, p. 154). No es así. Todo el análisis de Marx, a lo largo de cientos de páginas, distingue entre un valor de uso formal y un valor de uso particular. Más aún, no es que el análisis se refiera a distintos valores de uso particulares y concretos, sino, ante todo, al rol general de valor de uso. Sí hay, empero, dos valores de uso particulares que se miran con atención: el del metal precioso y el de la fuerza de trabajo.

De hecho, la principal distinción conceptual del valor de uso, que distingue el funcionamiento formal de la existencia particular, aparece al exponer la emergencia del oro-dinero como equivalente general. Se trata de un desdoblamiento. Este valor de uso desdoblado corresponde a un objeto que, por ciertas características de su corporeidad real, empieza a asumir funciones "formales" (Marx 1981; versión en alemán, 1962). 
Del dinero como simple medio de circulación se puede decir que deja de ser mercancía (mercancía particular) en tanto su material es indiferente y él satisface solo las necesidades del cambio, con exclusión de toda otra necesidad inmediata. Por otra parte, [...] el dinero es [...] mercancía universal en su forma pura, indiferente a su particularidad natural y por ello también a todas las necesidades inmediatas. (Marx, 1971a, v. 1, p. 15o, versión en alemán, 1976, p. 141)

Encontramos acá el uso marcado del matiz conceptual "mercancía particular", que distingue, como principal determinación conceptual, entre un valor de uso particular, que es como aparece una cosa en el mercado para el usuario; y un valor de uso formal, "desdoblado", cuando la cosa que es soporte del dinero - oro o plata, en principioadquiere relaciones específicas de portador del equivalente general. Esta cuestión es la más trabajada, la que está más claramente planteada, y la que aparece en los textos que Marx dio a la imprenta.

¿Por qué el oro? 1. es relativamente escaso; 2 . casi nunca es combinable con otros elementos y se lo halla en estado puro; 3 . puede dividirse y volver a reunirse en un nuevo fundido (1971b, p. 157; versión en alemán, 1980a, p. 41). Este cuerpo cumple funciones de equivalente general por tener características físico-químicas específicas. Cuando funciona como equivalente general:

el valor de uso de la mercancía dineraria se desdobla [verdöppelt]. Al lado de su valor de uso particular [besondre] en cuanto mercancía -el oro, p. ej., sirve para tapar caries, como materia prima de artículos suntuarios, etcétera-adquiere un valor de uso formal que deriva de sus funciones sociales específicas. (Marx, 1981, p. 109-110; versión en alemán, 1962, p. 104)

El funcionamiento formal se distingue de la existencia particular. Este valor de uso desdoblado corresponde a un objeto que, por atributos corporales asume funciones "formales". El valor de uso formal es el que "no existe más que como soporte [Träger] del valor de cambio [...] no se relaciona con ninguna necesidad individual real [wirklich]" sino como equivalente general: dinero (Marx, 1980b, p. 76; versión en alemán, 1980c, p. 160).

El metal, en tanto dinero, es capaz de tener un valor de uso "abstracto y puramente social, [que emerge de su] función como medio de circulación. [Tiene un] valor de uso particular en cuanto metal" (Marx, 1971b, p. 132; versión en alemán, 1980a, p. 25), es así materia prima para la manufactura.

El "desdoblamiento" es el término hegeliano que encuentra Marx para nombrar a la investidura simbólica. Esa investidura excede al caso planteado por Marx, el del dinero: es propio de distintas situaciones que no tienen por qué ser dinerarias, ni siquiera mercantiles. Corresponde a las diversas investiduras o significados que un valor de uso puede recibir.

La forma de valor (valor de cambio) no es sino una forma antigua y consolidada de una de las tantas configuraciones simbólicas de una cadena significante como es la de la comparación de un valor de uso con otro. Vender es una de las formas posibles del uso. Es decir que en la cadena sintagmática, temporal y práctica, un significante (y valor de uso) como una bolsa de trigo, en una situación determinada, puede ir al granero, o a la cocina, o al molino, o a las manos de otro propietario. En la forma valor de cambio, siempre, los dos polos de la ecuación, el relativo y el equivalente, funcionan como valores de uso. Para una de las partes funcionará como valor de uso particular, para la otra, como valor de uso formal.

Por eso que el collage de Baudrillard (1972): "el valor de uso es al valor de cambio como el significante al significado" (p. 151) plantea mal el problema. Pues se esconde que el 
valor de cambio no es sino un tipo de vínculo, simbólica e históricamente determinado, entre dos valores de uso. Si un cuerpo, sonoro o visual, no puede encontrar lugar claro y distinto en una cadena sintagmática con sentido, no tendrá significado alguno, y no podrá funcionar como significante. Si un cuerpo, sea cual sea su materialidad, no puede encontrar lugar claro y distinto en una cadena sintagmática práctica con sentido, no puede tener valor de uso. Y si una de las partes de una posible transacción comercial considera que lo que se le ofrece no tiene valor de uso, tampoco se realizará valor de cambio alguno.

h) Nombre. Inscripción. Isaak Rubin (que era bien consciente de eso que Althusser llamó el problema de la "expresión" o "representación”), afirmaba.

\section{A primera vista, el valor parece ser simplemente una propiedad de las cosas. Cuando decimos: "una mesa redonda, de roble, pintada, cuesta 25 rublos, o tiene el valor de 25 rublos”, puede mostrarse que esta proposición brinda información sobre cuatro propiedades de la mesa. (Rubin, 1985, p. 121-122)}

Pero falta señalar que, en la descripción de esa cosa llamada mesa, falta una quinta propiedad: su nombre, el nombre "mesa", que la ubica, por género próximo y diferencia específica, en el mundo de las cosas. Un valor de uso, en tanto significante, es siempre un lexema. No hay valor de uso no nombrado. ${ }^{9}$

Cuando Althusser intentó plantear en 1966 una "teoría general del significante” buscó identificar, no sin dudas, los significantes específicos de cada nivel: los conceptos serían significantes de la ciencia; los morfemas, de la lengua; los fantasmas, significantes del inconsciente. No mencionaba para nada cuáles podrían ser los significantes fundamentales de la instancia del modo de producción o una formación social determinada (Althusser, 1993). Propongo aquí, como se ha visto, que los valores de uso son los significantes fundamentales del orden social.

En la Grecia de Filipo y Aristóteles la sandalia era un artefacto ágrafo: elaborada por un artesano, se vendía sin marca simbólica especial. En la Gran Bretaña victoriana los objetos ya estaban perdiendo ese carácter ágrafo: las primeras leyes británicas de marca o branding son contemporáneas a la redacción de El capital. ${ }^{10}$ Hoy en cambio, el calzado es un artefacto inscripto. Porta una marca registrada, tiene diversos nombres mediante los que se pretende diferenciarlo de otros artefactos vendidos bajo esa misma marca registrada. Y está envuelto en un sinnúmero de otras inscripciones con las que se garantizaría que cada zapatilla de un modelo determinado de una marca registrada alemana o estadounidense es igual a otra, aunque haya sido fabricada por niños de Vietnam y empacada en Brasil. Y que (contigüidad establecida por el bombardeo publicitario) sería la misma que viste un futbolista argentino que juega en España.

En su teoría del fetichismo, Marx (1981) comparaba la "forma fantasmagórica” y suprasensible derivada de dicho quid pro quo con la "región neblinosa del mundo religioso", pues allí los productos humanos "parecen figuras autónomas dotadas de vida propia, en relación unas con otras" (p. 89). De hecho, el programa de investigar las proteicas cadenas significantes que invisten al mundo de los valores de uso supone ver justamente cómo estas figuras cobran autonomía y se relacionan unas con otras.

Clarisse Herrenschmidt mostró la unidad del movimiento histórico efectivo desde la lengua escrita con el alfabeto, los números arábigos y el código informático. Y destaca el doble origen, como aspectos de un único fenómeno (verificado en Elam, cerca de Sumeria, hace cincuenta siglos) de las primeras formas contables conocidas (bullo de arcilla), que son también las primeras formas de escritura, aun antes de la consolidación de la escritura cuneiforme (Herrenschmidt, 2007).
9. Lévi-Strauss destacó, en páginas muy conocidas, la "lógica de lo concreto" del "pensamiento salvaje" que nombraba plantas y animales aparentemente sin “utilidad". Mas nos damos cuenta que nombrar un objeto ya es una forma de usarlo; nada puede insertarse regularmente en una cadena de usos prácticos si no está antes clasificado y nombrado (Lévi-Strauss, 2013, p. 576-582).

10. 1862: "Marchandise Marks Act"; 1875: "Trade Marks Registration Act" (Bently, 2008). Si “la marca artesanal medieval era, en esencia, una responsabilidad legal [liability], la marca registrada moderna es, sin dudas, un activo del propietario" (Frank Schechter, cit. por Hamilton y Lai, 1989, p. 254). Es destacable que en China la marca mercantil tuvo un desarrollo más complejo y más temprano que en Europa (Hamilton y Lai, 1989). 
11. "La mercancía como tal, —su particularidad-también es por ende un contenido indiferente [gleichgültiger], meramente fortuito, y en général imaginado [vorgestellter], que se sitúa al margen de la relación económica formal" (Marx, 1971a, vol. 1, p. 210; versión en alemán, 1976, p. 193).
Hacia el siglo VII a.C. empieza a consolidarse otro fenómeno doble: por un lado, el metal usado para el intercambio aparece por primera vez inscripto: la moneda acuñada. Por el otro, los fenicios crearon el simple y democrático alfabeto contemporáneo. Posteriormente, la numeración indo-árabe ingresó a Europa en el siglo X y se consolidó tras el 1200: el valor de posición de cada cifra define unidades, centenas, decenas, la escritura de la cantidad se independiza del alfabeto. Este largo proceso de inscripción social culmina cuando el número permite construir autómatas: lo que empezó en 1936 con la máquina de Turing condujo, de modo crecientemente acelerado, a los automatismos contemporáneos de las últimas dos o tres décadas, con la generalización del código numérico. Hoy letra, número y código están inscriptos en el cuerpo de los valores de uso; todas estas inscripciones terminan por reformular la existencia material de los valores de uso en tanto mercancías. Veintisiete siglos después de la moneda lidia hemos llegado a los valores de uso acuñados, es decir, inscriptos. Tienen envoltorio, marca registrada y código QR. La marca del valor de cambio aparece ya inscripta en el cuerpo del valor de uso. De sandalia ágrafa a sandalia inscripta, el mundo de los valores de uso ha hecho un inmenso tránsito. Además de un nombre genérico (mesa, o sandalia), hay otra serie de nombres: nombre del modelo, del fabricante - que es el nombre de su empresa - u otro avatar. Hasta el mismo valor de cambio (la cifra del precio) suele estar escrita en los envases de las mercancías, con números o con un código de barras. En su aparente inmediatez, los valores de uso aparecen ahora llenos de misterios teológicos y sutilezas metafísicas. La riqueza, en las sociedades en las que rige el modo de producción capitalista, se muestra hoy como un inmenso cúmulo de valores de uso acuñados.

i) Valores del uso. Usar un objeto es ubicarlo en una cadena significante. El valor del uso no existe sino en el recorrido de esa cadena. Podemos afirmar que en ese proceso del uso se verifican estos valores:

1) Un valor corporal. El valor de uso como cuerpo porta una serie de atributos materiales objetivos, que nunca forman un repertorio cerrado. Desde la creatividad cotidiana a la invención científica, la materia siempre puede usarse de nuevas maneras. Siempre hay un resto irreducible, sin simbolizar. Cada cuerpo permite reinvenciones, utilizaciones nuevas, bricolages.

2) Un valor imaginario. La relación dual, especular, constituida entre el usuario y el bien que quiere usar, constituye lo que se designa habitualmente como utilidad. Es el tipo de análisis que han mirado los economistas marginalistas con su "función de utilidad" - la utilidad reducida a su forma pobre, unilateral, cuantitativa. ${ }^{11} \mathrm{La}$ necesidad, la utilidad, no están en el valor de uso, están en aquello que el valor de uso, como significante, señala. Señal que sólo existe como tal si algún sujeto la lee y la hace propia. Un valor de uso representa a un sujeto en relación a otro valor de uso. Este valor imaginario tiende hoy a estar determinado por la situación mercantil.

3) Valores simbólicos: los que vienen de la posición que la cosa toma en su relación con otras cosas. Hay al menos dos tipos de relaciones de posición; al respecto, volvemos a pedir prestado a Saussure algunos de sus términos (Saussure, 1997, p. 170).

3.1) Valor sintagmático. Un momento en un proceso de trabajo; de un proceso de producción consumidora (consumo productivo, o bien, consumo recreativo). Es la unión de dos maderos la que da valor de uso al tornillo; el mueble le da valor de uso a la unión de las dos maderas. El té le da valor de uso al azúcar. La cena le otorga valor de uso al vino; la situación cotidiana le otorga valor de uso a la cena.

3.2) Valor asociativo: en el mundo de los objetos, el usuario optará - de acuerdo con la situación específica en cierto momento y en cierto lugar- por un tornillo, o bien por 
otro de distinto tamaño, u otro material; o bien por un clavo, o por un alambre. Por un malbec o un torrontés, por un vino añejado en roble o uno más simple y barato.

El precio es uno entre tantos atributos del valor de uso. En tal uso efectivo intervienen tanto las propiedades corporales del objeto como la convicción del usuario potencial acerca de dichas propiedades, y de su conveniencia para tal empleo, frente a las alternativas efectivas que se imponen. Una botella de vino torrontés, de la bodega tal, con la marca registrada tal, que se vende a un precio $x$, puede asociarse a otros torrontés que portan otras marcas, o bien a otros vinos blancos, o bien a otros vinos cuyo valor se aproxime (o no) al precio $x$. Así, un valor de uso es "el centro de una constelación, punto en el cual convergen" otros significantes "relacionados, cuya suma es indefinida" (Saussure, 1997, p. 174). ${ }^{12}$

En estos casos cotidianos no hay un sujeto que razona a cada paso que da. Si los valores de uso funcionan como cadenas significantes, operan en automatismos de repetición, conocidos por los psicoanalistas. Si esos automatismos existen, entonces serían fundamentales como mecanismo de reproducción social.

Hay, por último, efectos políticos que redundan en un régimen de consumo. La elaboración de un sistema que impone por fuerza el patrón oro es cosa distinta que los atributos físicos del metal. Esas imposiciones sociales de valores de uso como el oro, podrían verificarse en otras mercancías básicas - soja, petróleo. En las grandes mareas sociales que impusieron al café y al té como estimulante cotidiano - y no la yerba mate, o a la hoja de coca. En las contramareas que prohibieron el alcohol en Estados Unidos por casi quince años, y amenazaron la posición dominante del café, buscando prohibirlo. En las inmensas cadenas significantes que ponen a valores de uso, uno al lado del otro, diferenciados por marcas registradas, nombres, envases, colores y relatos publicitarios distintos, ante la misma mirada del consumidor en el supermercado. El enorme cúmulo de valores de uso del mundo moderno no solo tiene una función de utilidad (mirada neoclásica) y una materialidad corporal (mirada técnica). Tiene también otro tipo de fuerza material que deriva del movimiento de los encadenamientos significantes. La acumulación de valores de uso, sobreinscriptos, sobrenominados, forma cadenas significantes, con sus lógicas y sus automatismos de repetición.

\section{Observaciones finales}

El concepto de valor de uso no solo permite expresar y analizar los fenómenos de consumo, sino también los de producción. El punto de vista que busco desarrollar no se sitúa en la división entre consumo y producción, sino más bien en la unidad inmediata de producción y consumo - tal como fuera expresada en la "Introducción" de los Grundrisse-. Creo que el funcionamiento de los valores de uso opera al interior de los procesos concretos (en los que, o bien predomina el consumo, "producción consumidora"; o bien la producción, "consumo productivo"). Procesos concretos materiales y también simbólicos, en tanto las cadenas materiales del uso son cadenas significantes.

Un concepto renovado de valor de uso supone conservar el empleo ya conocido del término en la larga tradición de economía política crítica, pero teniendo en cuenta también el funcionamiento de los valores de uso como significantes. Tomar en cuenta la materialidad del significante quiere decir que una mirada simbólica de la vida social no tiene por qué estar reñida con el análisis materialista.

Lo que hasta ahora parece predominar en la mayoría de la producción antropológica, que restringe el concepto de valor de uso a un atributo de las sociedades en donde predomina la producción para el autoconsumo, hace olvidar que la producción capitalista

\author{
12. Es lo que afirma Saussure \\ acerca de la encrucijada de \\ relaciones asociativas que \\ pueden trazarse con respecto \\ a toda palabra: en el término \\ "enseñanza" se entrecruzan series \\ como "enseñar, enseñamos"; \\ “aprendizaje, educación”; o "pu- \\ janza, bonanza” (Saussure, 1997; \\ ejemplo adaptado al castellano).
}


13. Ver en particular la formulación más tardía de Saussure, el cuaderno Constantin (Saussure, 1993). también es producción de valores de uso - por ejemplo, que también el dinero tiene valor de uso-. En este marco destaca sólidamente el caso de Ben Fine, para quien el concepto de valor de uso es central. Buscando que el análisis del consumo no quede desgajado de sus determinaciones capitalistas, Fine objeta el posmodernismo fundado en Baudrillard, pues "tiene el efecto de desgajar el consumo de la economía, en tanto el consumo se vuelve (X-) construido [sic] como sistema de signos" (Fine, 2008, p. 68), de modo que el "consumo se vuelve simbolismo, puro y simple" (Fine, 2008, p. 73). Aunque creo que su punto de vista en general es muy atendible, estas páginas han buscado señalar qué vale la pena conservar de lo que él rechaza, en tanto la dimensión simbólica es parte constitutiva de toda relación social.

Por eso Saussure, pues, como Marx, hizo una revolución epistemológica al correr de lugar lo que habitualmente se considera "material" o "inmaterial" o ideal. Marx criticó el materialismo precedente, desde sus tesis Ad Feuerbach, por ver a la realidad efectiva como un objeto ajeno a la vida sensorial subjetiva y activa. Así como criticó los análisis "idealistas" por dejar de lado la materialidad histórica y cotidiana. Como sugirió Étienne Balibar (haciendo un contrapunto crítico hacia Hannah Arendt), Marx trastocó las relaciones precedentes entre praxis y poiēsis (Balibar, 1995). Quiero destacar que la operación de Saussure fue análoga y comparable. Cambió la historia de la lingüística al replantear el lugar material de las partes constituyentes a las dos caras del signo. El signo, hasta entonces, vinculaba un nombre (ideal) a una cosa (material). ${ }^{13}$ Desde Saussure, lo que antes era un nombre es una imagen acústica; lo que antes una cosa es ahora un concepto. El hecho de enfocar el vínculo entre un segmento sonoro y un concepto trastoca e invierte el lugar que hasta entonces tenían la materialidad y la idealidad. Ese reacomodamiento inauguró buena parte del pensamiento contemporáneo.

\section{Agradecimientos}

Estas reflexiones sintetizan mucho tiempo de elaboración. No he tenido financiación especial para escribir estas líneas, aunque su investigación y su redacción han sido parte de mis labores como docente e investigador en el sistema universitario público argentino, en Buenos Aires y en Río Negro. Quiero reconocer la muy atenta y paciente lectura anónima que ayudó a disminuir los defectos de esta redacción y los errores formales en las referencias. Estoy agradecido a Étienne Balibar, que siempre me alentó a insistir con esta línea de análisis. (Y recordar también a Juan Carlos Marín y a León Rozitchner, porque los extraño). Agradezco también a Hugo Ratier y a Horacio González, que se interesaron en publicar las primeras formulaciones de este punto de vista. Y a Hugo Trinchero y demás miembros de un espacio desde el cual tratamos de abrir un campo de docencia e investigación en antropología económica en la Universidad de Buenos Aires; en particular a Liliana Landaburu, que pervive en mi memoria por su buen humor, por su inteligencia y por su calidad humana. 


\section{Q Referencias bibliográficas}

» Abduca, R. (2004). ¿Qué es un valor de uso? Producción, significante, enunciación. El Ojo Mocho, Revista de Crítica Política y Cultural, no 18-19, 39-43.

"Abduca, R. (2005). Consumo y subjetividad: el valor de uso como significante. Etnia, no 46-47, 7-26.

» Abduca, R. (2010). Acerca del concepto de valor de uso: signo, consumo y subjetividad. La hoja de coca en la Argentina. (tesis de doctorado), Universidad de Buenos Aires, Facultad de Filosofía y Letras, Ciudad Autónoma de Buenos Aires, Argentina.

» Abduca, R. (2018). Valeur d'usage et signifiant. Notes pour une théorie unifiée. Actuel Marx, 64, 146-161.

» Althusser, L. (1993 [1966]). Trois notes sur la théorie des discours. En Écrits sur la psychanalyse: Freud et Lacan (pp. 111-170). Paris: Stock/IMEC.

" Aristóteles (1959). Politics. Edic. bilingüe. London: Heinemann, Loeb Classical Library.

"Balibar, É. (1995). The Philosophy of Marx. London: Verso.

" Baudrillard, J. (1972). Pour une critique de l'économie politique du signe. Paris: Gallimard.

»Bently, L. (2008). The Making of Modern Trade Marks Law: The Construction of the Legal Concept of Trade Mark (1860-80). En L. Bently, J. Davis y J. C. Ginsburg, Trade Marks and Brands (pp. 3-41). Cambridge: Cambridge University Press.

"Chevalier, J. (1982). Civilization and the Stolen Gift: Capital, Kin, and Cult In Eastern Peru. Toronto: University of Toronto Press.

"Fine, B. (2008). The World of Consumption. The Material and Cultural Revisited. London: Routledge.

» Goux, J.-J. (1973) [orig. 1969]. Los equivalentes generales en el marxismo y el psicoanálisis. Buenos Aires: Caldén.

》 Graeber, D. (2011). Debt. The First 5000 Years. New York: Melville.

》 Gregory, C. A. (1982). Gifts and Commodities. London, Academic Press.

" Gregory, C. A. (1997). Savage Money. The Anthropology and Politics of Commodity Exchange. Amsterdam: Harwood.

» Hahn, C. y Hart, K. (2011). Economic Anthropology. History, Ethnography, Critique. Cambridge: Polity.

» Hamilton, G-G. y Chi-Kong Lai (1989). Consumerism without Capitalism. Consumption and Brand Names in Late Imperial China. En H. J. Rutz y B. S. Orlove, The Social Economy of Consumption (pp. 253-280). Lanham: University Press of America.

» Herrenschmidt, C. (2007). Les trois écritures. Langue, signe, code. Paris: Gallimard.

» Jevons, W. S. (2013). The Theory of Political Economy. New York: Palgrave-Macmillan.

" Lacan, J. (1966a). Le séminaire sur «La Lettre-volée». En Écrits, (pp. 11-61), Paris: Seuíl.

» Lacan, J. (1966b). L'instance de la lettre dans l'inconscient, ou la raison depuis Freud. En Écrits, (pp. 493-598), Paris: Seuíl.

"Lévi-Strauss, C. (1977). Apéndice de los capítulos III y IV. En Antropología estructural (pp. 74-88). Buenos Aires: Eudeba. 
» Lévi-Strauss, C. (1980). “Introduction a l'œuvre de Marcel Mauss”. M. Mauss, Sociologie et anthropologie (pp. ix-lii). Paris: P.U.F.

» Lévi-Strauss, C. (2013). OEuvres. Paris: Gallimard, Bibliothèque de la Pléiade.

» Marx, K. (1962). Das Kapital. Libro I; Marx-Engels Werke, t. 23, Berlin: Dietz.

» Marx, K. (1971a). Elementos fundamentales para la crítica de la economía política (Grundrisse) 1857-1858 (vols. 1-3), Buenos Aires: Siglo XXI

» Marx, K. (1971b). "Fragmento de la versión primitiva de la 'Contribución a la crítica de la economía política”. Elementos fundamentales para la crítica de la economía política (Grundrisse) 1857-1858 (vol. 3, pp. 119-218). Buenos Aires: Siglo XXI.

» Marx, K. (1976). Grundrisse der Kritik der politischen Ökonomie; Ökonomische Manuskripte und Schriften 1858-1861. MEGA, 2º Abteilung, t. 1; Berlin: Dietz.

» Marx, K. (1980a). “Urtext «Zur Kritik»”, Ökonomische Manuskripte und Schriften 1858-1861, (pp. 17-94). MEGA, 2을 Abteilung, t. 2; Berlin: Dietz.

» Marx, K. (1980b). Contribución a la crítica de la economía política. México: S. XXI.

» Marx, K. (1980c). Zur Kritik der Politischen Ökonomie. Ökonomische Manuskripte und Schriften 1858-1861, (pp. 95-245). MEGA, 2º Abteilung, t. 2; Berlin: Dietz.

» Marx, K. (1981). El capital. Tomo I / Libro primero (vols. 1-3). México: Siglo XXI.

» Menger, C., (1996). Principios de economía política. Barcelona: Folio.

» Narotzky, S. (1997). New Directions of Economic Anthropology. London: Pluto.

» Ricardo, D. (2004). On the Principles of Political Economy and Taxation. (Ed. Sraffa-Dobb). Indianapolis: Liberty Fund.

» Robotham, D. (2005). Political Economy. En J. Carrier (ed.), Handbook of Economic Anthropology (pp. 41-58). Cheltenham: Elgar.

» Roseberry, W. (1993). Anthropologies and Histories. London: Routledge.

»Sahlins, M. (1972). Stone Age Economics. Chicago: Aldine.

»Saussure, F. de (1993). Troisième cours de linguistique générale (1910-11) d'après les cahiers d'Émile Constantin. Edic. de E. Komatsu. Oxford: Pergamon.

» Saussure, F. de (1997). Cours de linguistique générale. Edic. de T. de Mauro. Paris: Payot.

» Taussig, M. (1980). The Devil and Commodity Fetishism in South America. Chapel Hill: University of Carolina Press. 